3

4

\section{Individual listening success explained by synergistic interaction of two distinct neural filters}

*Author correspondence:

Sarah Tune, Jonas Obleser

Department of Psychology

University of Lübeck

Maria-Goeppert-Str. 9a

23562 Lübeck

Email: sarah.tune@uni-luebeck.de; jonas.obleser@uni-luebeck.de

Running title: Neural tracking and alpha power in attentive listening

Number of words in Abstract: 148

Number of words in Introduction: 848

Number of words in Discussion: 1939

34 pages, 5 Figures, 0 Tables, Includes Supplemental Information

Conflict of Interest: The authors declare no competing financial interests.

Keywords EEG, alpha power lateralization, neural speech tracking, speech processing, auditory selective attention 
Neural tracking and alpha power in attentive listening

\section{Abstract}

38 Successful speech comprehension requires the listener to differentiate relevant

39 from irrelevant sounds. Recent neurophysiological studies have typically

40 addressed one of two candidate neural filter solutions for this problem: the

41 selective neural tracking of speech in auditory cortex via the modulation of

42 phase-locked cortical responses, or the suppression of irrelevant inputs via alpha

43 power modulations in parieto-occipital cortex. However, empirical evidence on

44 their relationship and direct relevance to behavior is scarce. Here, a large, age-

45 varying sample $(\mathrm{N}=76,39-70$ years) underwent a challenging dichotic listening

46 task. Irrespective of listeners' age, measures of behavioral performance, neural

47 speech tracking, and alpha power lateralization all increased in response to

48 spatial-attention cues. Under most challenging conditions, individual listening

49 success was predicted best by the synergistic interaction of these two distinct

50 neural filter strategies. Trial-by-trial fluctuations of lateralized alpha power and

51 ignored-speech tracking did not co-vary, which demonstrates two

52 neurobiologically distinct filter mechanisms. 


\section{Introduction}

Real-life listening is characterized by the concurrence of sound sources that compete for our attention (Cherry, 1953). Successful speech comprehension thus relies on the selective filtering of relevant and irrelevant inputs. How does the brain instantiate attentional filter mechanisms that allow for the controlled inhibition and amplification of speech? Recent neuroscientific approaches to this question have focused on two different neural filter strategies originating from distinct research traditions:

From the visual domain stems an influential line of research that suggests a domain-general role of parieto-occipital alpha-band ( 8-12 Hz) oscillatory activity in implementing selective attention. Here, the key assumption is that increases in alpha power support the controlled, top-down suppression of behaviorally-irrelevant information (Rihs et al., 2007; Jensen and Mazaheri, 2010; Foxe and Snyder, 2011; Händel et al., 2011). Accordingly, listening tasks that require spatially-directed attention are neurally supported by a hemispheric lateralization of changes in alpha power over occipital, parietal but also sensory cortices (Kerlin et al., 2010; Müller and Weisz, 2011; Ahveninen et al., 2013; Wöstmann et al., 2016; Tune et al., 2018; Wöstmann et al., 2018).

In addition, there is a prominent line of research that focuses on the role of low-frequency $(1-8 \mathrm{~Hz})$ neural activity in and around auditory cortex in the selective representation of sound inputs. It is assumed that slow cortical dynamics temporally align with (or "neurally track") auditory input signals to allow for optimal processing of behaviorally-relevant sensory information (Schroeder and Lakatos, 2009; Schroeder et al., 2010; Henry and Obleser, 2012). In human speech comprehension, recent studies found evidence for the preferential neural tracking of attended compared to ignored speech in superior temporal brain areas close to auditory cortex (Ding and Simon, 2012; Mesgarani and Chang, 2012; Horton et al., 2013; Zion Golumbic et al., 2013; O'Sullivan et al., 2014).

However, with few exceptions, these two proposed neural filter strategies have been studied independently of one another (but see Kerlin et al., 2010). Also, they have often been studied using tasks that are difficult to relate to natural listening situations (Lakatos et al., 2016; Henry et al., 2017). We thus lack understanding whether or how modulations in (lateralized) alpha power, presumably arising from domain-general networks involving parietal cortex, and the neural tracking of attended and ignored speech in wider auditory cortex interact in the service of successful listening behavior. At the same time, few studies using more real-life listening and speech-tracking measures were able to explicitly address the functional relevance of the discussed neural filter strategies, that is, their immediate consequences for speech comprehension success (but see Mesgarani and Chang, 2012).

In the present EEG study, we aim at closing these gaps by leveraging the statistical power and representativeness of a large, age-varying participant sample. We use a novel dichotic listening paradigm to enable a synoptic look at concurrent changes in alpha power and neural speech tracking at the single-trial level. More specifically, our linguistic variant of a classic Posner paradigm (Posner, 1980) emulates a challenging dual-talker listening situation in which 
103 speech comprehension is supported by two different listening cues (Alavash et 104 al., 2018). These cues encourage the use of two complementary cognitive 105 strategies to improve comprehension: A spatial-attention cue guides auditory 106 attention in space, whereas a semantic cue affords more specific semantic 107 predictions of upcoming speech. Previous research has shown that the sensory 108 analysis of speech and, to a lesser degree, the modulation of alpha power are 109 influenced by the availability of higher-order linguistic information (Obleser and 110 Weisz, 2012; Sohoglu et al., 2012; Peelle et al., 2013; Presacco et al., 2016a; 111 Wöstmann et al., 2017).

112 Both cues were presented in an informative and uninformative version, 113 allowing us to examine relative changes in listening success and in the 114 modulation of neural measures thought to enable auditory selective attention. 115 Based on the neural and behavioral results, we focused on four research 116 questions (see Fig. 1).

First, we predicted that informative listening cues should increase speech comprehension success: These cues allow to deploy auditory selective attention (compared to divided attention), and to generate more specific (compared to only general) semantic predictions, respectively.

Secondly, we asked how the different cue-cue combinations would modulate the two key neural measures-alpha power lateralization and neural speech tracking. We hypothesized that selective (compared to divided) attention should increase the strength of alpha power lateralization and neural tracking of the to-be-attended speech signal, respectively.

An important and often neglected third research question pertains to a direct, trial-by-trial relationship of these two candidate neural measures: Do changes in alpha power lateralization impact the degree to which attended and ignored speech signals are neurally tracked by low-frequency cortical responses?

Our most important final research question asked whether the observed (co-)variation of alpha power and neural speech tracking of attended and ignored speech would in turn allow us to predict single-trial behavioral success in this challenging listening situation. In addressing these research questions, we acknowledge additional, potentially detrimental influences on listening cess and its supporting neural strategies. These include age, hearing loss, or 137 hemispheric asymmetries in speech processing due to the well-known right-ear 138 advantage (Kimura, 1961; Broadbent and Gregory, 1964). 
bioRxiv preprint doi: https://doi.org/10.1101/512251; this version posted January 4, 2019. The copyright holder for this preprint (which was not certified by peer review) is the author/funder, who has granted bioRxiv a license to display the preprint in perpetuity. It is made available under aCC-BY-NC-ND 4.0 International license.

Neural tracking and alpha power in attentive listening

139

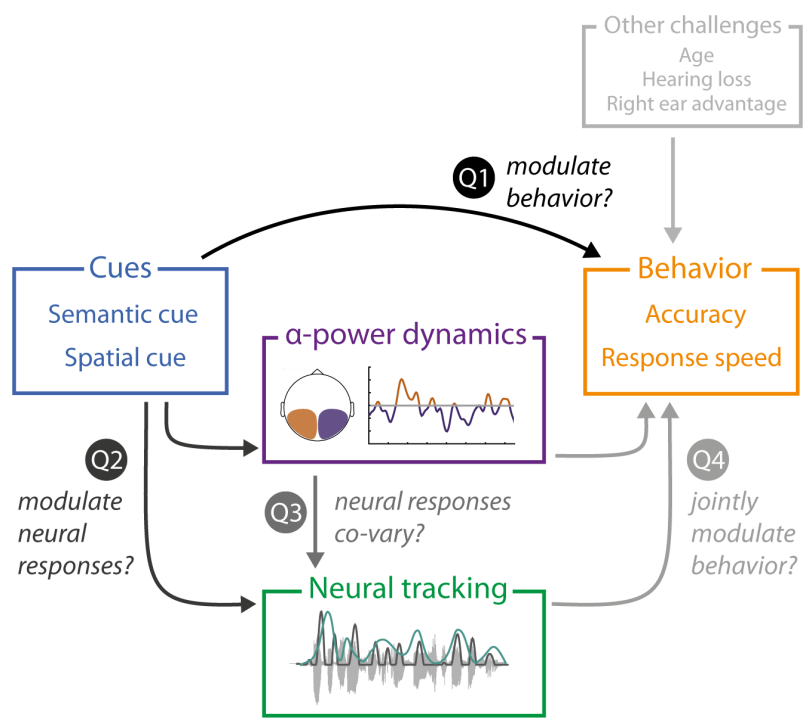

Figure 1. Schematic illustration of the research questions addressed in the present study. The dichotic listening task manipulated the attentional focus and semantic predictability of upcoming input using two separate visual cues. We investigated whether informative cues would enhance behavioral performance (Q1). In line with (Q2), we also examined the degree to which listening cues modulated the two neural measures of interest: neural tracking and lateralization of alpha power. Finally, we assessed (Q3) the co-variation of neural measures, and (Q4) their potency in predicting behavioral performance. Furthermore, we controlled for additional factors that may challenge listening success and its underlying neural strategies. 
bioRxiv preprint doi: https://doi.org/10.1101/512251; this version posted January 4, 2019. The copyright holder for this preprint (which was not certified by peer review) is the author/funder, who has granted bioRxiv a license to display the preprint in perpetuity. It is made available under aCC-BY-NC-ND 4.0 International license.

Neural tracking and alpha power in attentive listening

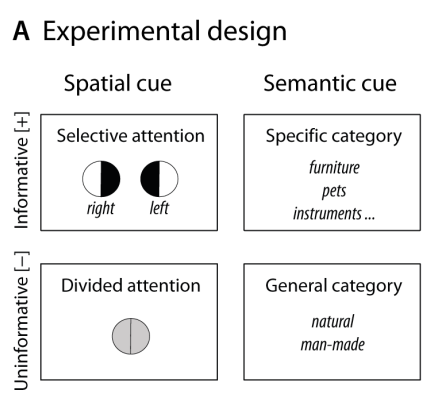

C Informative cues improve behavior

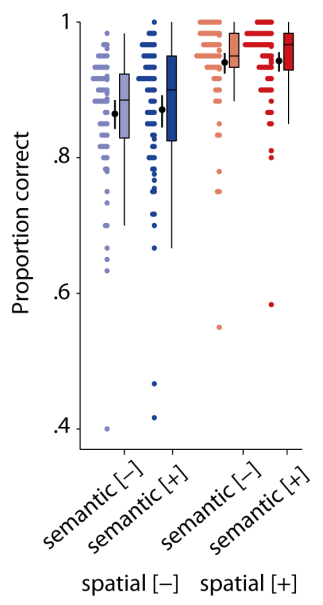

\section{B Trial structure}

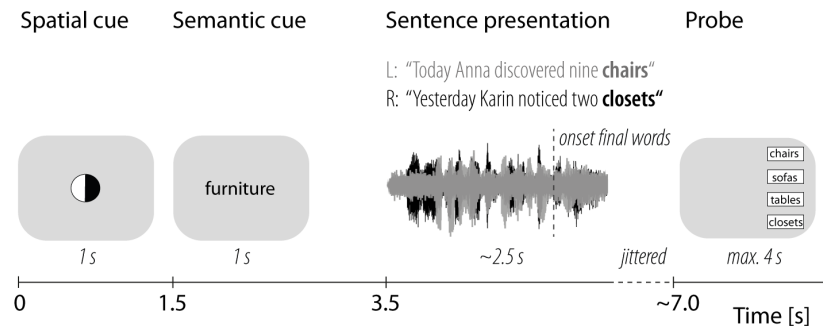

D Individual cue benefit
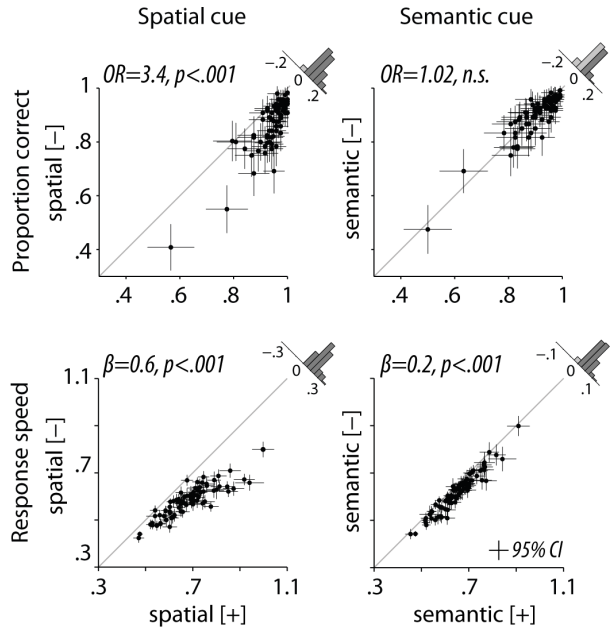

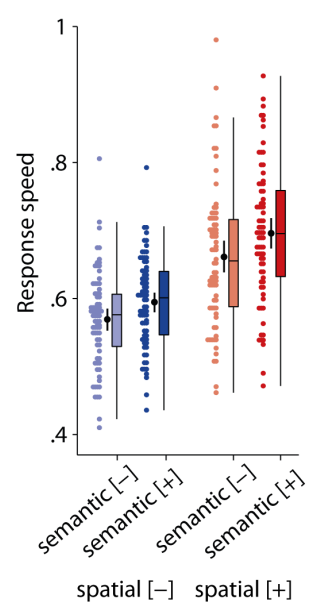

Figure 2. Experimental design and behavioral benefit from informative cues.

(A) Visualization of the employed $2 \times 2$ design. The level of the spatial and semantic cue differed on a trial-by-trial basis. Top row shows the informative [+] cue levels, bottom row the uninformative [-] cue levels.

(B) Schematic representation of the temporal order of events for a given trial. Successive display of the two visual cues precedes the dichotic presentation of two sentences uttered by the same female talker. After sentence presentation, participants had to select the final word from four alternative words.

(C) Grand average and individual results of accuracy and response speed shown per cue-cue combination. Colored dots are individual $(\mathrm{N}=76)$ trial-averages, black dots and vertical lines show individual means \pm bootstrapped $95 \%$ confidence intervals.

(D) Individual cue benefits for all 76 participants displayed separately for the two cues and dependent behavioral measures, respectively. Across participants, we observed a consistent cue benefit of the informative compared to the uninformative spatial-attention cue on both accuracy and response speed. Black dots indicate individual trial-averages \pm bootstrapped $95 \%$ confidence intervals. Histograms show the distribution of the difference for informative vs. uninformative levels. OR: Odds ratio parameter estimate from generalized linear mixed-effects models; $\beta$ : slope parameter estimate from general linear mixed-effects models.

The following figure supplements are available for figure 2:

Figure supplement 1. Histogram showing age distribution of $\mathrm{N}=76$ participants across six age bins.

Figure supplement 2. Individual and mean air-conduction thresholds (PTA) averaged across the left and right ear.

Figure supplement 3. Right-ear advantage for accuracy and response speed. 
Neural tracking and alpha power in attentive listening

\section{Results}

We recorded EEG from an age-varying sample $(\mathrm{N}=76)$ of healthy middle-aged and older adults (39-70 years of age) who performed a challenging dichotic listening task (Alavash et al., 2018). In this linguistic variant of a classic Posner paradigm, participants listened to two competing sentences spoken by the same female talker, and were asked to identify the final word in one of the two sentences. Importantly, sentence presentation was preceded by two visual cues. First, a spatial-attention cue encouraged the use of either selective or divided attention by providing informative or uninformative instructions about the tobe-attended, and thus later probed, ear. The second cue indicated the semantic category that applied to both final target words. The provided category could represent a general or specific level, thus allowing for more or less precise prediction of the upcoming speech signal (Fig. 2A, B).

Our analyses aim at explaining behavioral task performance in a challenging listening situation, and the degree to which it is leveraged by two key neural measures of auditory attention: the lateralization of 8-12 Hz alpha power, and the neural tracking of attended and ignored speech by slow cortical responses. Using generalized linear mixed-effects models on single-trial data, we investigated the cue-driven modulation of behavior and neural measures, as well as the interaction of neural measures, and their (joint) influence on listening success.

\section{Informative spatial cue improves listening success}

The analysis of behavioral performance tested the effect of informative versus uninformative listening cues on speech comprehension success. Overall, participants achieved a mean accuracy of $90.5 \%$ (sd 7.6\%) but as shown in Fig. $2 \mathrm{C}$, the behavioral results varied between the different combination of listening cues. We investigated these relative changes in accuracy and response speed at the single-trial level. As highlighted above, our models consider the potential influence of additional factors such as the probed ear (left vs. right), age, or hearing acuity as measured by the pure-tone average across both ears (PTA; see also Fig. 2-supplements 1 and 2). Variability between participants (see Fig. 2D) and items was accounted for via the inclusion of participant- and item-specific random effects (see Tables S1 and S2 for full model details).

As expected, the analyses revealed a strong behavioral benefit of informative compared to uninformative spatial-attention cues. In selectiveattention trials, participants responded more accurately and faster (accuracy: odds ratio $(O R)=3.37, p<.001$; response speed: $\beta=.58, p<.001)$. That is, when cued to one of the two sides, participants responded on average $250 \mathrm{~ms}$ faster and their probability of giving a correct answer increased by $5 \%$.

Participants also responded generally faster in trials in which they were given a specific and thus informative semantic cue $(\beta=.19, p<.001)$, most likely reflecting a semantic priming effect that led to faster word recognition. A corresponding semantic-cue effect on accuracy was only found for trials in which the onset of the later-probed sentence preceded that of the unprobed sentence (interaction semantic cue $\times$ first onset: $O R=2.29, p<.001$; simple effect semantic cue (probed-first trials): $O R=1.59, p=.03$ ). Note that these slight time 
Neural tracking and alpha power in attentive listening

213 delays between dichotically presented sentences resulted from these sentences

214 being naturally recorded but subsequently onset-aligned for the task-relevant,

215 sentence-final words (see Supplemental Information for details).

216 As in a previous fMRI implementation of this task (Alavash et al., 2018),

217 we did not find evidence for any interactive effects of the two listening cues on

218 either accuracy or response speed. The inclusion of the respective interaction

219 terms either did not significantly improve model fit (likelihood ratio test;

220 accuracy: $X^{2}=.04 ; p=.84$ ) or did not reach significance in the final best-fitting

221 model (response speed: $\beta=.10, p=.13$ ).

222 
bioRxiv preprint doi: https://doi.org/10.1101/512251; this version posted January 4, 2019. The copyright holder for this preprint (which was not certified by peer review) is the author/funder, who has granted bioRxiv a license to display the preprint in perpetuity. It is made available under aCC-BY-NC-ND 4.0 International license.

Neural tracking and alpha power in attentive listening

A Spatio-temporal dynamics of alpha power modulation

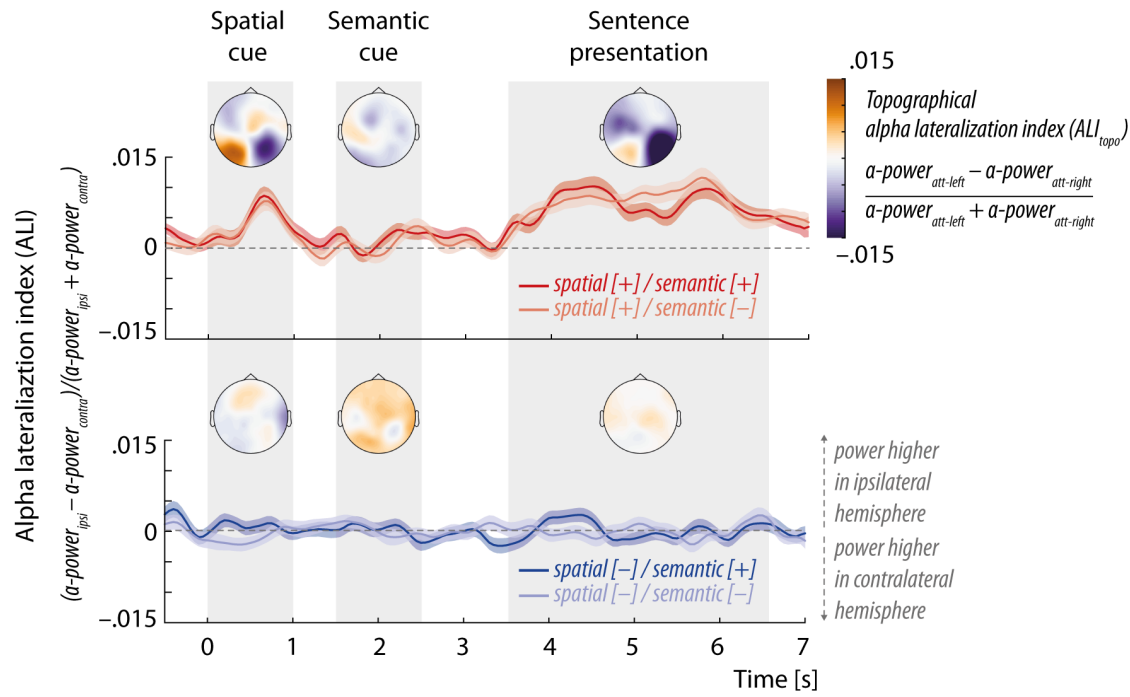

B Spatial attention increases alpha power lateralization

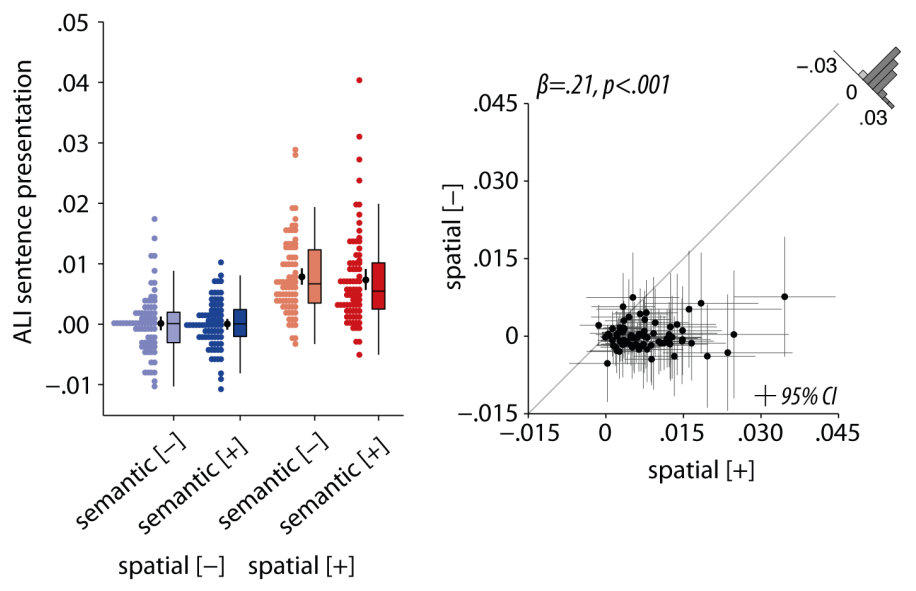

Figure 3. Informative spatial cue elicits increased alpha power lateralization before and during speech presentation. (A) Attentional modulation of $8-12 \mathrm{~Hz}$ alpha power shown throughout the trial across all $\mathrm{N}=76$ participants. Topographical maps indicate the spatial distribution of the alpha lateralization index (ALItopo) collapsed across the semantic-cue levels in three time windows of interest (spatial cue: $0-1 \mathrm{~s}$; semantic cue: 1.5-2.5 s; sentence presentation: 3.5-6.5 s; shaded in grey). Warm colors indicate higher power in attend-/probed-left compared to attend-/probed-right trials. The opposite relationship holds for cold colors. Red and blue traces show the temporally resolved alpha lateralization index (ALI) for each cue-cue combination. Positive values indicate relatively higher alpha power in the hemisphere ipsilateral to the attended/-probed sentence compared to the contralateral hemisphere.

(B) Strength of the ALI during sentence presentation (3.5-6.5 s) shown per cue-cue combination (right plot). Colored dots represent trial-averaged individual results, black dots and error bars indicate the grand-average and bootstrapped 95\% confidence intervals. Consistent increase in alpha power lateralization across all participants (left plot). Black dots represent individual mean ALI values \pm bootstrapped $95 \%$ confidence intervals. Histogram shows distribution of differences in ALI for informative vs. uninformative spatial-cue levels. $\beta$ : slope parameter estimate from the corresponding general linear mixed-effects model.

The following figure supplements are available for figure 3:

Figure supplement 1. Whole-trial overall oscillatory power averaged across all trials, electrodes, and $\mathrm{N}=76$ participants.

Figure supplement 2. Grand-average whole-trial alpha lateralization index (ALI) calculated without adjustment for overall differences in power across hemispheres.

Figure supplement 3. Grand-average whole-trial alpha lateralization index (ALI) time-locked to the onset of the sentence-final target words. 
250 Lateralization of alpha power modulated by selective attention but not 251 semantic predictability

252 In line with our second research question, we tested the hypothesis that an 253 informative spatial cue would lead to a lateralization of alpha power in parietal254 occipital brain areas. We relied on two established neural indices (Haegens et al., 255 2011; Mesgarani and Chang, 2012): The topographical alpha lateralization index $256\left[\mathrm{ALI}_{\text {topo }}=\left(\mathrm{a}-\right.\right.$ power $_{\text {att-left }}-\mathbf{a}$-power $\left.{ }_{\text {att-right }}\right) /\left(\mathbf{a}-\right.$ power $_{\text {att-left }}+\alpha-$ power $\left.\left._{\text {att-right }}\right)\right]$ contrasts 257 alpha power in attend-left vs. attend-right trials (for divided-attention 258 conditions: probed-left vs. probed-right trials) and yields a topographical 259 representation of attention-related changes in power. It is complemented by the 260 alpha lateralization index $\left[\mathrm{ALI}=\left(\mathbf{a}-\right.\right.$ power $_{\text {ipsi }}-\mathrm{a}$-power $\left._{\text {contra }}\right) /\left(\mathbf{a}-\right.$ power $_{\text {ipsi }}+\mathbf{a}$ 261 power contra $_{\text {] }}$ ] that compares alpha power ipsi- and contralateral to the 262 attended/probed ear to provide a temporally-resolved single-trial measure of 263 alpha power lateralization (for overall whole-trial changes in power, see Fig. 3supplement 1).

In accordance with the proposed role of alpha power modulation in supporting selective attention, the instruction to pay attention to one of the two sides elicited a pronounced lateralization of $8-12 \mathrm{~Hz}$ alpha power over posterior channels during the spatial cue and during the actual sentence presentation (see $\mathrm{ALI}_{\text {topo }}$ maps in Fig. $3 \mathrm{~A}$ ). The time course of alpha power lateralization per cue-cue combination further supported this observation: We found a strong but transient increase in lateralization in response to an informative spatial-attention cue. After a subsequent break-down of the lateralization during semantic-cue presentation, it re-emerged in time for the start of dichotic sentence presentation and peaked again during the presentation of the task-relevant sentence-final words (see Fig. 3-supplement 3 for ALI time course time-locked to the presentation of sentence-final words).

To investigate the influence of the spatial-attention but also the semantic cue on the degree of alpha power lateralization during sentence presentation in more detail, we extracted single-trial average values from this time window and submitted them as dependent measure to linear mixedeffects analysis (see Fig. 3B and Table S3 for full model details). As expected, the analysis revealed a highly significant and consistent increase in alpha power lateralization for informative compared to uninformative spatial cues $(\beta=.21$, $p<.001)$. Notably, we did not find any evidence of a modulation of alpha lateralization during sentence presentation by the semantic cue nor any joint influence of the spatial and semantic cue (likelihood ratio tests; main effect semantic cue: $X^{2}{ }_{1}=.32, p=.57$; interaction of cues: $X^{2}{ }_{1}=.19, p=.67$ ). Note that the analysis of alpha power lateralization during spatial-cue presentation yielded a comparable increase in lateralization in response to an informative spatial cue $290 \quad(\beta=.08, p<.001$; see Table S4 for full details). 
bioRxiv preprint doi: https://doi.org/10.1101/512251; this version posted January 4, 2019. The copyright holder for this preprint (which was not certified by peer review) is the author/funder, who has granted bioRxiv a license to display the preprint in perpetuity. It is made available under aCC-BY-NC-ND 4.0 International license.

Neural tracking and alpha power in attentive listening

A Envelope reconstruction models

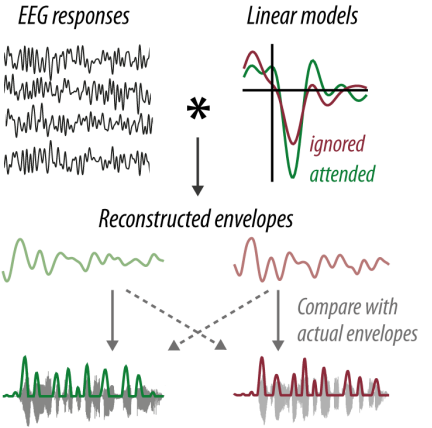

C Neural tracking of attended envelope

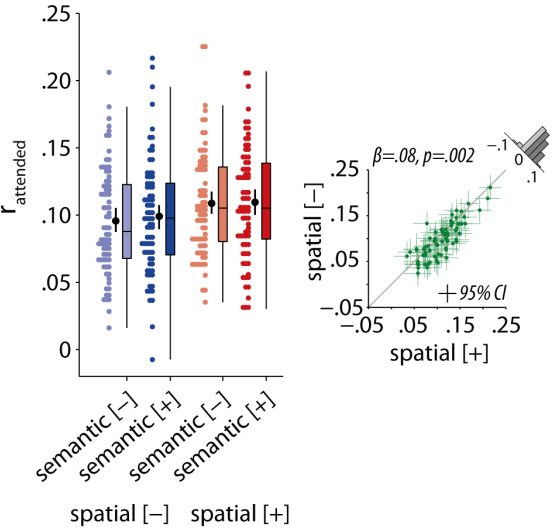

B Encoding of attended and ignored envelope

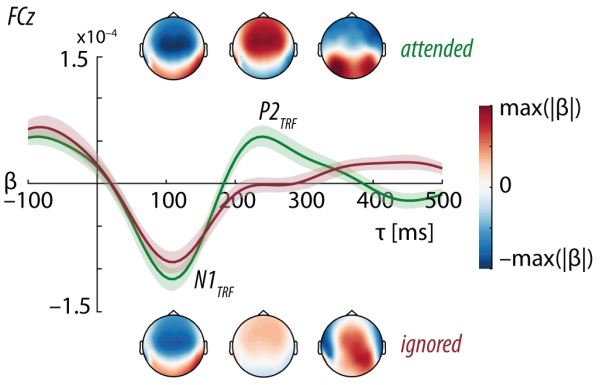

D Neural tracking of ignored envelope

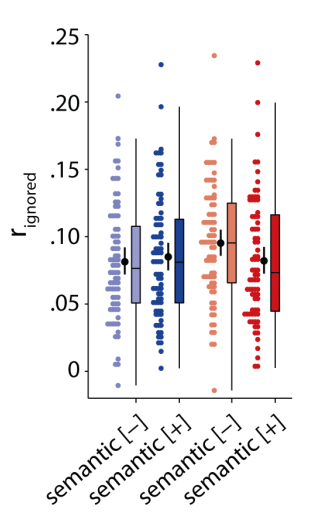

spatial [-] spatial [+]

Figure 4. Neural speech tracking of attended and ignored sentences.

(A) Schematic representation of the linear backward model approach used to reconstruct onset envelopes on the basis of recorded EEG responses. Following the estimation of linear backward models (see Fig. 4-supplement 1 for details), envelopes of attended and ignored sentences were reconstructed via convolution of EEG responses with the estimated linear backward models at all EEG channels. Reconstructed envelopes were compared to the envelopes of presented sentences to assess reconstruction accuracy.

(B) Topographical distribution of linear backward model weights shown in line with forwardtransformed temporal response functions for attended (green, top row) and ignored (red, bottom row) speech at electrode $\mathrm{FCZ}$. Models are averaged across all $\mathrm{N}=76$ participants, and across attendleft and attend-right trials. Topographical maps correspond to the time lag intervals of 90-110 ms, 200-250 ms, and 350-450 ms. Error bands indicate 95\% confidence intervals.

(C, D) Changes in neural tracking of attended and ignored speech across cue-cue combinations. Colored dots represent trial-averaged individual results, black dots and error bars indicate the grand-average and bootstrapped $95 \%$ confidence intervals. Neural tracking of attended speech is stronger under selective attention (C, right plot). Neural tracking of ignored speech is modulated by two-way interactions of spatial cue $\times$ semantic cue, and spatial cue $\times$ probed ear ( $D$, right plots). Colored dots represent trial-averaged correlation coefficients per participant. Error bars show bootstrapped $95 \%$ confidence intervals.

The following figure supplements are available for figure 4:

Figure supplement 1. Training and testing of envelope reconstruction models.

Figure supplement 2. Encoding of attended and ignored speech shown separately per probedear condition.

Figure supplement 3. Neural tracking strength per reconstruction model and attention condition. Figure supplement 4. Decoding accuracy in selective-attention trials. 
Neural tracking and alpha power in attentive listening

\section{Selective tracking of attended and ignored speech}

In close correspondence with our analysis of alpha power lateralization, we investigated whether changes in attentional demand and semantic predictability would modulate the neural tracking of attended and ignored speech. To this end, we used linear backward ('decoding') models to reconstruct the onset envelopes of the to-be-attended and -ignored sentences (for simplicity hereafter referred to as attended and ignored). We compared the reconstructed envelopes to the envelopes of presented sentences using Pearson's correlation. The resulting correlation coefficients are a measure of reconstruction accuracy and serve as an indicator of how strongly the envelopes of attended and ignored sentences were tracked by slow cortical responses (i.e., neural tracking; see Fig. 4A and figure supplement 1). Reconstruction models were trained on selective-attention trials, only, but then utilized to reconstruct attended (probed) and ignored (unprobed) envelopes for both attention conditions (see Methods for details).

Figure $4 \mathrm{~B}$ shows the topographical distribution of regression weights obtained from the linear backward models temporally aligned with the most prominent deflections in the forward-transformed temporal response functions (TRFs) of attended and ignored speech. We averaged models across all $\mathrm{N}=76$ participants, and across attend-left (i.e., ignore-right) and attend-right (i.e., ignore-left) trials (see Fig. 4-supplement 2 for model results split by probed ear).

Both representations show a similar encoding of the attended and ignored sentences in very early stages of processing $(<150 \mathrm{~ms})$ as indicated by comparable regression weights over fronto-central channels and corresponding TRF deflections in the time window covering the $\mathrm{N} 1_{\mathrm{TRF}}$ component. By contrast, during ensuing processing stages reflected by the $\mathrm{P} 2_{\mathrm{TRF}}$ component, there are clear differences observable in the strength of neural tracking: For the attended sentence, we observed a pronounced $\mathrm{P} 2_{\mathrm{TRF}}$ component and the absence thereof for the ignored sentence. In addition, in later processing stages (> 300ms), deflections in the TRF for attended and ignored speech follow trajectories of opposite polarity and the associated topographical maps of backward model weights suggest the involvement of distinct brain networks. A complementary analysis examining models results split by probed ear, revealed that this pattern was particularly pronounced in attend-left/ignored-right trials (see Fig. 4supplements $2 A, D$ ).

\section{Listening cues influence the tracking of attended and ignored speech}

To gain a more differentiated understanding of how selective attention and semantic predictability influence the selective cortical representation of speech signals at the single-trial level, we focused on the neural tracking strength of the attended and ignored sentence. More specifically, our linear models predicted the strength of single-trial correlations between the reconstructed and presented envelopes (see Fig. 4-supplement 3 for neural tracking strength per reconstruction model and attention condition).

For selective-attention trials, we observed a degree of neural tracking (attended: mean $r=.11$, range: .003-.22; ignored: mean $r=.09$, range: .007-.23) that was similar in magnitude to that reported in recent neural speech tracking 
369 studies using linear backward models (e.g., O'Sullivan et al., 2014; Crosse et al., 370 2015). However, as shown in Figure $4 C$ and $D$, the neural tracking of attended 371 and ignored sentences varied across cue-cue combinations. We statistically 372 assessed these cue-driven modulations in two separate linear mixed-effects 373 models (see Tables S5 and S6 for full model details). Note that for sake of 374 consistency, for divided-attention trials, we refer to the reconstruction of the 375 probed envelope as the attended envelope, and to the reconstruction of the 376 unprobed envelope as the ignored envelope despite the absence of corresponding instructions.

Giving credence to the suggested role of selective neural speech tracking as a neural filter strategy, we found stronger neural tracking of the attended envelope following an informative spatial-attention cue as compared to an uninformative one $(\beta=.08, p=.002)$. The semantic predictability of the sentence-final words, however, did not modulate the neural tracking of the attended envelope (likelihood ratio test; $X^{2}{ }_{1}=.69, p=.41$ ).

Notably, the analysis of ignored-speech tracking revealed a qualitatively different pattern of results: Here, neural tracking strength was modulated by the interactive effect of the spatial-attention cue together with either the semantic cue or the probed ear (spatial cue $\times$ semantic cue: $\beta=-.12, p=.02$; spatial cue $\times$ probed ear: $\beta=-.08, p=.006$ ). Following an informative spatial-attention cue, we found increased neural tracking of the ignored sentence when less prior information about the upcoming input was available, that is, when it was preceded by an uninformative semantic cue (simple effect semantic cue, informative spatial cue: $\beta=-.08, p=.01$; uninformative spatial cue: $\beta=.03, p=.36$ ). At the same time, when participants had to pay attention to both sides, taskirrelevant sentences played to the left ear were tracked more strongly than taskirrelevant sentences played to the right ear (simple effect probed ear, selective: $\beta=.04, p=.13$; divided: $\beta=.12, p<.001$ ).

\section{Decoding accuracy}

Given that our challenging listening task presented two concurrent sentences that were (i) of short duration, (ii) spoken by the same female talker, (iii) highly similar with respect to their onset envelopes, and (iv) presented against speechshaped noise, we wished to further evaluate the potency of the employed envelope reconstruction approach under such difficult conditions. To this end, we examined the decoding accuracy across participants in selective-attention trials (see also Fig. 4-supplements 1, 4 and 5). Evaluated at the single-trial level, attention was correctly decoded if the envelope reconstructed by the attended reconstruction model was more similar to the attended envelope than to the ignored envelope ( $\left.r_{\text {attended }}>r_{\text {ignored }}\right)$ and vice versa for the ignored reconstruction model.

$410 \quad$ Unsurprisingly, the achieved decoding accuracy was lower than that 411 reported in speech tracking studies using much longer segments of continuous 412 speech but still reached a mean decoding accuracy of $55 \%$ (range: $0.43-0.65$ ) for 413 the reconstruction model of the attended envelope and 48\% (range: $0.36-0.72$ ) 414 for the reconstruction model of the ignored envelope (see Etard et al., 2018 for 415 decoding accuracy as a function of stimulus duration). Importantly, when 
416 investigated at the single-subject level, the attended reconstruction model 417 yielded decoding accuracies that exceeded the empirical chance level of $58 \%$ in 41820 out of 76 participants. The ignored reconstruction model yielded significantly 419 above chance performance in 10 out 76 participants. In addition to the 420 aforementioned experimental conditions that created a particularly difficult 421 scenario for stimulus reconstruction, it is important to bear in mind that 422 decoding accuracy is not only influenced by specifics of the linear model itself 423 but also by how closely participants followed the spatial-cue instructions. For 424 these reasons, our analyses capitalized on the single-trial neural tracking 425 strength as a more direct and fine-grained measure of how strongly the 426 attended and ignored sentence were cortically represented in a particular trial.

\section{Additional influences on behavioral performance and neural measures}

Our modelling approach accounted for a number of additional factors known to challenge speech comprehension, and to modulate neural signature of attention (see Fig. 1). We observed that participants' performance varied in line with the well-attested right-ear advantage (REA, also referred to as left-ear disadvantage) in the processing of linguistic materials (Kimura, 1961; Broadbent and Gregory, 1964). More specifically, participants responded both faster and more accurately (response speed: $\beta=.06, p<.001$; accuracy: $O R=1.38, p<.001$ ) when they were probed on the last word presented to the right compared to the left ear. While we observed differences in the strength of ignored-speech tracking across probed-left and probed-right trials under the more challenging divided-attention condition (see section above), the degree of alpha power lateralization did not change with the probed ear $(\beta=.03, p=.66)$.

With respect to age and hearing acuity, we observed that decreases in hearing acuity (PTA) led to less accurate response $(O R=.75, p=.007)$, whereas increased age only led to overall slower $(\beta=-.12, p=.003)$ but not less accurate responses $(O R=.89, p=.29)$. In addition, and in accordance with previous reports (Presacco et al., 2016b), we observed a significant increase in neural tracking of the attended sentence for older participants $(\beta=.06, p=.02)$. Again, the strength of alpha power lateralization did not co-vary with participants' age $(\beta=-.01$, $p=.39$ ), confirming results of a previous dichotic listening EEG study on a subsample of the present cohort (Tune et al., 2018).

Lastly, the neural tracking of the attended envelope was stronger when its presentation began prior to that of the ignored envelope $(\beta=.33, p<.001)$,

\section{Independent single-trial modulation of alpha power lateralization and neural} 456 tracking

457 Our third major goal was to investigate whether the modulation of alpha power 458 and neural tracking strength reflect two dependent neural strategies of 459 attention at all. To this end, using two single-trial linear mixed-effects models, 460 we tested whether the neural tracking of the attended and ignored sentence 
461 could be predicted from alpha power dynamics (here, the alpha power at 462 electrodes ipsi- and contralateral to the focus of attention/probed ear, that is, 463 the relative signal modulations that constitute the single-trial alpha 464 lateralization index). We reasoned that it is the up-regulation of contralateral 465 alpha power in the service of selective inhibition, and the down-regulation of 466 ipsilateral alpha power in the service of controlled amplification that in turn 467 might facilitate the differential tracking of attended and ignored speech (cf. Fig. $4685 A)$.

469 However, neither the tracking of the attended envelope (main effect 470 alpha ipsi: $\beta=-.01, p=.36$; main effect alpha contra: $\beta=.01, p=.32$; interaction ipsi $471 \times$ contra: $\beta=-.001, p=.54)$ nor the tracking of the ignored envelope could be 472 predicted by changes in ipsi- or contralateral alpha power or by their interaction 473 (main effect alpha ipsi: $\beta=-.005, p=.71$; main effect alpha contra: $\beta=.002, p=.88$; 474 interaction ipsi $\times$ contra: $\beta=-.001, p=.64$; see Tables $S 6$ and $\mathrm{S7}$ for full model 475 details).

476 This notable absence of an alpha lateralization-speech tracking 477 relationship was consistent across the spatial-attention and probed-ear 478 conditions (i.e., inclusion of the respective terms did not improve model fit; all ps $479>0.2$ ). Importantly, the observed independence of these two neural measures 480 also did not hinge on whether we modeled the change in alpha power as two 481 separate regressors or included the strength of the alpha lower lateralization 482 expressed by the ALI instead (see Table S9 for details). 
bioRxiv preprint doi: https://doi.org/10.1101/512251; this version posted January 4, 2019. The copyright holder for this preprint (which was not certified by peer review) is the author/funder, who has granted bioRxiv a license to display the preprint in perpetuity. It is made available under aCC-BY-NC-ND 4.0 International license.

Neural tracking and alpha power in attentive listening

A Hypothesized relationship

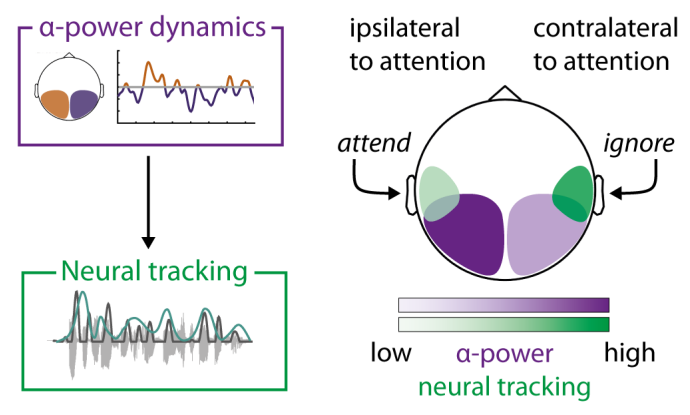

B Independence of neural measures

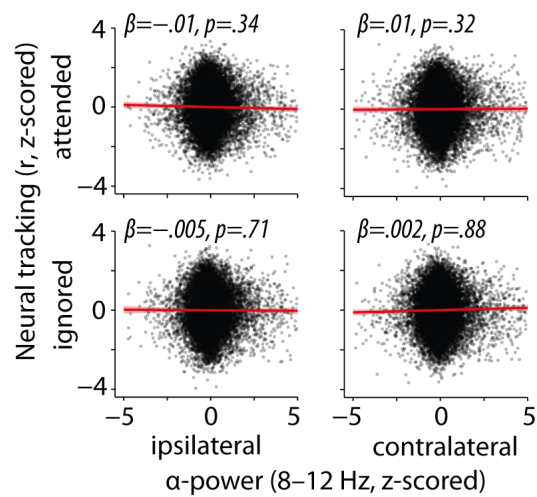

C Neural measures jointly modulate accuracy

Left ear probed
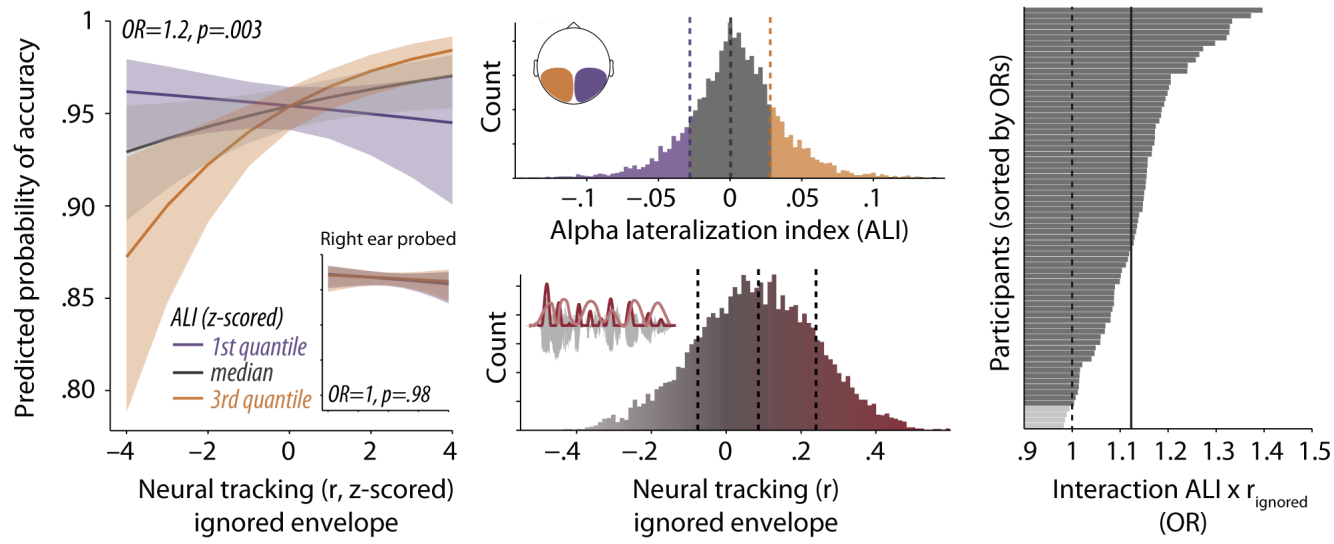

(OR)

Figure 5. Relationship of alpha power dynamics and neural tracking and their synergistic influence on behavior.

(A) Hypothesized inverse relationship of changes in alpha power and neural tracking in the hemisphere contra- and ipsilateral to the focus of attention. Changes in alpha power are assumed to drive changes in neural tracking strength. Schematic representation of expected co-variation in the two neural measures during an attend-left trial.

(B) Independence of changes in neural tracking and alpha power during sentence processing as revealed by two separate linear mixed-effects models predicting the neural tracking strength of attended and ignored speech, respectively. Error band denotes $95 \%$ confidence interval. Black dots represent single-trial observed values.

(C, left panel) Visualization of the interactive effect of alpha power lateralization (ALI) and neural tracking of the ignored envelope as predicted from the generalized linear model for probed-left trials. Inset shows absence of the effect in probed-right trials. Neural measures are z-scored across all $\mathrm{N}=76$ participants. The relationship of neural tracking and accuracy is shown for three quantiles of the ALI. Error bars and bands represent $95 \%$ confidence intervals. (C, middle panel) Distribution of ALI (top) and neural tracking raw values (bottom) prior to z-scoring. Vertical dashed lines index the median, first and third quantiles. (C, right panel) Consistency of the two-way interaction of ALI and neural tracking across all 76 participants. Effects for individual participants are estimated via the inclusion of corresponding random slopes in a model of probed-left trials, only. Solid black line represents the fixed-effects level interaction effect; black dotted line indicates an odds ratio (OR) of 1. Dark grey bars are individual subjects with an OR above 1; light grey bars show subjects with an OR of below 1.

The following figure supplements are available for figure 5 :

Figure supplement 1 . Modulation of response speed by neural tracking strength ( $\left.r_{\text {attended }}\right)$ per attention condition. 
512 Neural strategies of attention jointly support listening success under 513 challenging conditions

514 Having established the surprising functional independence of alpha power

515 lateralization and speech tracking, the final and most important piece of our

516 investigation becomes in fact statistically more tractable: If alpha power

517 dynamics in parieto-occipital cortex, and neural speech tracking in auditory

518 cortices essentially act as two independent neural filter strategies, we can

519 proceed to probe their relative functional relevance for behavior in a factorial-

520 design fashion. That is, we were interested in how far changes in listening

521 success related to the independent (i.e., as main effects) or joint influence (i.e., as

522 an interaction) of neural measures. We answered these question using the same

523 linear mixed-effects models as in testing our first research question (Q1 in Fig.1),

524 as well as the influence of additional factors (see Tables S1 and S2 for details).

525 First, neural tracking of the attended envelope had opposing effects on

526 listeners' response speed, depending on the attention condition (interaction

527 neural tracking $\times$ spatial cue: $\beta=-.04, p=.004)$ : In the behaviorally more difficult

528 divided-attention condition, increased tracking of the to-be-attended sentence

529 led to faster responses $(\beta=.02, p=.01)$. In cued selective-attention trials, we found

530 only a weaker trend, in the opposite direction $(\beta=-.02, p=.1$; see Fig. 5-

531 supplement 1 ).

532 Secondly, for response accuracy as our most important indicator of

533 listening success, we also found a beneficial effect of stronger neural tracking

534 that occurred under the behaviorally more challenging condition: Specifically,

535 we observed a joint influence of the degree of alpha power lateralization and

536 ignored-speech tracking when the less favorable left ear was probed (interaction

537 neural tracking ignored $\times \mathrm{ALI} \times$ probed ear: $O R=.86, p=.03$; simple effects neural

538 tracking ignored - left ear: $O R=1.17, p=.003$; right ear: $O R=1, p=.98$ ).

Probed-left trials substantially increased the probability of giving a

540 correct answer for neural states of co-occurring strong alpha power

541 lateralization and strong ignored-speech tracking (Fig. 5C). This particular brain-

542 behavior relationship was highly consistent across the large sample of

543 participants, and we observed a corresponding effect in 72 out of 76

544 participants.

545 Moreover, this joint influence of alpha power lateralization (ALI) and

546 neural tracking was specific to their joint occurrence in the time window of

547 sentence presentation. We did not observe any comparable relationship of ALI

548 and neural tracking on behavior when we substituted the degree of alpha

549 power lateralization during sentence presentation for the degree of

550 lateralization during the spatial-attention cue (see Tables S10 and S11 for

551 details).

\section{Discussion}

554 In the present study, we utilized the power of a large, representative sample of

555 middle-aged and older listeners to explicitly address the question of how two

556 different neural filter strategies, typically studied in isolation, jointly shape

557 listening success. In our dichotic listening task, we recorded the 
558 electroencephalogram and observed systematic changes in alpha power 559 lateralization and the neural tracking of attended speech in response to an 560 informative spatial-attention cue. These results not only provide unprecedented 561 large-sample support for their suggested roles as neural signatures of selective 562 attention. We also were able to address two important but still insufficiently 563 answered questions: How do the two neural filter strategies relate to one 564 another, and how do they impact speech comprehension success in a 565 demanding real-life listening situation?

First, when related at the single-trial, single-subject level, we found the modulation of the two neural measures to be statistically independent, 568 underlining their functional segregation and speaking to two distinct neurobiological implementations.

Secondly, the presence of an informative spatial-attention cue not only boosted both neural measures of interest but also consistently boosted 572 behavioral performance. However, changes at the neural and at the behavioral 573 levels were not generally related. Instead, we observed a joint influence of alpha 574 power lateralization and ignored-speech tracking on the behavioral outcome 575 only for trials in which the slightly speech-disadvantaged left ear was probed.

\section{Alpha power and neural speech tracking implement independent neural filter strategies}

579 Attention-related changes in alpha power dynamics and in the neural tracking 580 of speech signals arise from distinct cortical areas; involve neurophysiological 581 signals operating at different frequency regimes; and are typically investigated 582 in separate lines of research. Nevertheless, there is preliminary evidence 583 suggesting that these two neural filter strategies may exhibit a systematic 584 relationship (Kerlin et al., 2010; Lakatos et al., 2016; Wöstmann and Obleser, 585 2016; Henry et al., 2017; Tune et al., 2018). Therefore, in the present study we probed the hypothesis that a domain-general, top-down controlled attentional filter implemented via regulation of alpha power in parieto-occipital cortices would in turn facilitate the selective sensory analysis of speech signals in auditory cortex (Schroeder and Lakatos, 2009; Schroeder et al., 2010).

However, our analyses of trial-by-trial variation in alpha power and neural tracking of attended and ignored speech revealed that the relative up- or down-regulation of alpha power varied independently of the degree to which the attended or the ignored sentence were neurally tracked in auditory cortex. Therefore, the present results speak against a consistent, linear relationship of neural filter strategies. We see instead the coexistence of two complementary but independent neural solutions to the implementation of auditory selective attention for the purpose of speech comprehension.

The proposed independence of neural filters in the current task is supported by additional pieces of evidence: On the one hand, the two neural measures were differentially affected by additional factors modelled in our analyses: The strength of alpha power lateralization was only influenced by the spatial-attention cue. By contrast, and in line with previous reports, the degree spatial-attention and semantic cue, participants' age, as well as the probed ear 
605 (Sohoglu et al., 2012; Peelle et al., 2013; Presacco et al., 2016a; 2016b). These 606 results corroborate the assumption of separate cortical circuits of distinct 607 bottom-up and top-down communication that give rise to the neural filter 608 strategies under investigation here.

609 Further support stems from a recent fMRI study from our laboratory that 610 used the same dichotic listening task and tested a subsample of the present 611 participant cohort (Alavash et al., 2018). In a graph-theoretic analysis of cortex612 wide networks, the reconfiguration of resting state networks in adaptation to 613 the listening task did not increase integration of larger brain networks. Instead it 614 prioritized local processing by means of higher segregation. Crucially, switching 615 from rest to task triggered the emergence of an auditory control sub-network 616 that enabled a fine-tuned cross-talk between auditory, temporal, and frontal 617 control areas. That is, we observed a reconfigured composition of brain 618 networks that involved brain areas generally associated with the neural tracking 619 of speech but not those typically engaged in the controlled inhibition of 620 irrelevant information via top-down regulation of alpha power (Banerjee et al., 621 2011; Nourski and Brugge, 2011; Giraud and Poeppel, 2012; Sadaghiani and 622 Kleinschmidt, 2016).

623 What can be concluded about the functional interplay of the two neural 624 filter strategies when put in context of previous electrophysiological studies 625 reporting diverging results (Kerlin et al., 2010; Lakatos et al., 2016; Henry et al., 626 2017)? Taken together, the results do provide strong evidence in favor of a generally existent functional tradeoff between attentional filter mechanisms. However, we suggest that the precise nature of this interplay of attentional filter mechanisms hinges on a number of factors such as the particular task demands, and potentially on the level of temporal and/or spatial resolution at which the two neural signatures are examined (Lakatos et al., 2016).

\section{Synergistic interaction of neural filter strategies supports speech} comprehension under challenging conditions

635 With the present study, we explicitly addressed the often overlooked question 636 of how trial-by-trial changes at the neural level would impact behavior, here 637 single-trial speech comprehension success (van Ede et al., 2012; Ding and Simon, 638 2014; Krakauer et al., 2017). Notably, despite an unprecedentedly large sample 639 of almost eighty listeners, adequate number of within-subject trials, and a 640 sophisticated linear-model approach, we did not find evidence of a direct (i.e. 641 independent of the listening cues or related factors such as the probed ear) 642 influence of the relative strength of alpha power lateralization or of the neural 643 tracking of the attended or ignored sentence.

644 Instead, for trials in which the left ear was probed, we observed a 645 significant modulation of accuracy that depended on the relative levels of both 646 alpha power lateralization and the neural tracking of the ignored sentence. 647 Highly consistent across individuals, in neural states of strong alpha 648 lateralization a sizable performance difference arises from the degree of neurally 649 tracking the ignored speech signal: There was a predicted 20-\% increased 650 chance of accurately responding in a trial, as ignored-speech tracking moved 651 from weakest to strongest. 
In light of the well-established right ear advantage (Kimura, 1961; Broadbent and Gregory, 1964), observing such an intricate interaction pattern should come as no surprise to psychoacousticians, psychologists, and neuroscientists alike. Nevertheless, the dependence on probed ear is an exploratory finding and begs replication in the second half of our large-scale, ongoing cohort acquisition (intended total $\mathrm{N}$ is 160).

Despite the exploratory nature of this last finding, the elaborate circumstances under which the significant influence of neural changes on behavior became manifest, highlight the complexity of often implicitly assumed brain-behavior relationships and their dependence on a multitude of contextual factors. Moreover, even in cases where a consistent relationship of attentionrelated neural fluctuations and a behavioral outcome can be established, the neural contribution to behavioral change may be surprisingly low (as shown for alpha power modulation before; van Ede et al., 2012).

Indeed, in previous studies on alpha power lateralization, in particular those that focused on middle-aged and older adults, evidence for the behavioral importance of this neural signature has been mixed (Sander et al., 2012; Hong et al., 2015; Mok et al., 2016; Leenders et al., 2018). The present results are generally in line with one of our own recent studies carried out on a subsample of the present cohort. Using a fine-grained analysis of concurrent neural and behavioral changes, we found only a weak link between the strength of alpha lateralization and accuracy in a similar dichotic listening task (Tune et al., 2018).

Given the research field's present focus on highly naturalistic designs (Hamilton and Huth, 2018), such as the concurrent presentation of two narratives, evidence on the functional importance of selective neural speech tracking is generally sparse (Power et al., 2012; Zion Golumbic et al., 2013; O'Sullivan et al., 2014; Fiedler et al., 2017; 2019). A notable exception is the study by Mesgarani and Chang (2012) who observed an enhanced auditory cortical representation of the attended versus ignored talker in correct compared to error trials. Further supporting the idea that the behavioral importance of neural filter strategies may depend on the level of task difficulty, overall performance in this task was notably lower ( $70 \%)$ than in the present study.

Consequently, a testable hypothesis arises: The more attentionally demanding a listening situation is, the more will listening success depend on the fidelity of neural filter strategies and their potential interaction. The present data hold initial evidence for this assumption: The overall good behavioral performance (around 90\%) attests that our task had a moderate level of difficulty. The joint influence of the lateralized alpha power and ignored-speech speech tracking on accuracy occurred only under the relatively more demanding condition, that is, when the behaviorally disadvantaged left ear was probed.

\section{Neural tracking of irrelevant speech is not irrelevant to listening success}

A crucial finding of the present study lies in the behavioral importance of the degree to which the ignored sentence was neurally tracked. This finding adds to a growing body of evidence suggesting that the successful deployment of auditory selective attention depends on the neural fate of both to-be-attended 
and to-be-ignored speech signals (e.g. Melara et al., 2002; Chait et al., 2010; for review see Shinn-Cunningham, 2008, Shinn-Cunningham and Best, 2008; Kaya and Elhilali, 2017).

Our results are generally compatible with the findings of a recent, source-localized EEG study (Fiedler et al., 2019) providing evidence for the active suppression of to-be-ignored speech signals via an enhanced cortical representation of the ignored talker. Importantly, this amplification in the neural representation of to-be-ignored speech in areas beyond auditory cortex was only found in situations where the distracting talker was louder and thus perceptually more dominant.

In absence of any systematic manipulation of the signal-to-noise ratio (SNR) of attended and ignored speech, the trial-by-trial differences in the probed ear employed here may be likened to a shift in the subjectively perceived SNR. That is, the observed right ear benefit for both accuracy and response speed may in part be driven by the greater difficulty of tuning out the to-be-ignored sentence when it was played to the right compared to the left ear.

Our data provide preliminary support for this assumption of a differential neural representation of ignored speech presented to the right versus the left ear (see Fig. 4-supplements 2A, D): In attend-left/ignore-right trials, we observed more selective cortical representations of attended and ignored speech, crucially supported by a late distinct response to the ignored speech signal. While additional research should systematically test the hypothesis put forward here, our results suggest that under relatively challenging listening situation (e.g., when the behaviorally disadvantages left ear is probed), listening success increasingly depends on the selective neural encoding of both attended and ignored speech.

\section{Conclusion}

In a large, representative sample of adult listeners, we have provided evidence that single-trial listening success in a challenging, dual-talker acoustic environment will not be meaningfully modelled when focusing on a single, oneto-one neural substrate alone: An attentional cue increases the engagement of two distinct neural filter strategies and boosted listening success. However, there was no direct link between behavioral and neural changes. Instead, the observed joint influence of alpha power lateralization and ignored-speech tracking in trials where the slightly speech-disadvantaged left ear was probed highlights the intricate nature of this brain-behavior relationship and should temper over-simplified accounts of the predictive power of neural filter strategies for behavior. It also emphasizes a third major finding of this study, potentially disruptive to ongoing research programs in decoding attentional states from the listening brain: There is behavioral relevance to the degree of neurally representing ignored speech in the cortical response. 


\section{Materials and Methods}

\section{Participants and procedure}

Seventy-six right-handed German native speakers (median age 56 years; range 39-70 years; 28 males; see Fig. 1-supplement 1 for age distribution) were included in the sample. All participants are part of a large-scale study on the neural and cognitive mechanisms supporting adaptive listening behavior in healthy middle-aged and older adults ("The listening challenge: How ageing brains adapt (AUDADAPT)"; https://cordis.europa.eu/project/rcn/197855_en.html). Handedness was assessed using a translated version of the Edinburgh Handedness Inventory (Oldfield, 1971). All participants had normal or corrected-to-normal vision, did not report any neurological, psychiatric, or other disorders and were screened for mild cognitive impairment using the German version of the 6-Item Cognitive Impairment Test (6CIT; Jefferies and Gale, 2013).

As part of our large-scale study on adaptive listening behavior in healthy aging adults, participants also underwent a session consisting of a general screening procedure, detailed audiometric measurements, and a battery of cognitive tests and personality profiling (see Tune et al., 2018 for details). This session always preceded the EEG recording session. Only participants with normal hearing or age-adequate mild-to-moderate hearing loss were included (see Fig. 1-supplement 2 for individual audiograms). Participants gave written informed consent and received financial compensation ( $8 €$ per hour). Procedures were approved by the ethics committee of the University of Lübeck and were in accordance with the Declaration of Helsinki.

\section{Dichotic listening task}

In a novel linguistic variant of a classic Posner paradigm (Posner, 1980), participants listened to two competing, dichotically presented sentences. They were probed on the sentence-final word in one of the two sentences. Critically, two visual cues preceded auditory presentation. First, a spatial-attention cue either indicated the to-be-probed ear, thus invoking selective attention, or did not provide any information about the to-be-probed ear, thus invoking divided attention. Second, a semantic cue specified a general or a specific semantic category for the final word of both sentences, thus allowing to utilize a semantic prediction. Cue levels were fully crossed in a $2 \times 2$ design and presentation of cue combinations varied on a trial-by-trial level (Fig. 2A). The trial structure is exemplified in Figure 2B. Details on stimulus construction and recording can be found in the Supplemental Information.

Each trial started with the presentation of a fixation cross in the middle of the screen (jittered duration: mean $1.5 \mathrm{~s}$, range 0.5-3.5 s). Next, a blank screen was shown for $500 \mathrm{~ms}$ followed by the presentation of the spatial cue in the form of a circle segmented equally into two lateral halves. In selective-attention trials, one half was black, indicating the to-be-attended side, while the other half was white, indicating the to-be-ignored side. In divided-attention trials, both halves appeared in grey. After a blank screen of 500 ms duration, the semantic cue was presented in the form of a single word that specified the semantic category of both sentence-final words. The semantic category could either be 
given at a general (natural vs. man-made) or specific level (e.g. instruments, fruits, furniture) and thus provided different degrees of semantic predictability. Each cue was presented for $1000 \mathrm{~ms}$.

After a $500 \mathrm{~ms}$ blank-screen period, the two sentences were presented dichotically along with a fixation cross displayed in the middle of the screen. Finally, after a jittered retention period, a visual response array appeared on the left or right side of the screen, presenting four word choices. The location of the response array indicated which ear (left or right) was probed. Participants were instructed to select the final word presented on the to-be-attended side using the touch screen. Among the four alternative were the two actually presented nouns as well as two distractor nouns from the same cued semantic category.

Stimulus presentation was controlled by PsychoPy (Peirce, 2007). The visual scene was displayed using a 24" touch screen (ViewSonic TD2420) positioned within an arm's length. Auditory stimulation was delivered using inear headphones (EARTONE $3 \mathrm{~A}$ ) at sampling rate of $44.1 \mathrm{kHz}$. Following instructions, participants performed a few practice trials to familiarize themselves with the listening task. To account for differences in hearing acuity within our group of participants, individual hearing thresholds for a 500-ms fragment of the dichotic stimuli were measured using the method of limits. All stimuli were presented $50 \mathrm{~dB}$ above the individual sensation level. During the experiment, each participant completed 60 trials per cue-cue condition, resulting in 240 trials in total. The cue conditions were equally distributed across six blocks of 40 trials each $(\sim 10 \mathrm{~min})$ and were presented in random order. Participants took short breaks between blocks.

\section{EEG data acquisition and preprocessing}

Participants were seated comfortably in a dimly-lit, sound-attenuated recording booth where we recorded their EEG from 64 active electrodes mounted to an elastic cap (Ag/AgCl; ActiCap / ActiChamp, Brain Products, Gilching, Germany). Electrode impedances were kept below $10 \mathrm{k} \Omega$. The signal was digitized at a sampling rate of $1000 \mathrm{~Hz}$ and referenced on-line to the left mastoid electrode (TP9, ground: AFz).

For subsequent off-line EEG data analyses, we used the EEGlab (version 14_1_1b; Delorme and Makeig, 2004) and Fieldtrip toolboxes (version 2016-0613; Oostenveld et al., 2011), together with customized Matlab scripts. Independent component analysis (ICA) using EEGlab's default runica algorithm was used to remove all non-brain signal components including eye blinks and lateral eye movements, muscle activity, heartbeats and single-channel noise. Prior to ICA, EEG data were re-referenced to the average of all EEG channels (average reference). Following ICA, trials during which the amplitude of any individual EEG channel exceeded a range of 200 microvolts were removed.

\section{Behavioral data analysis}

We evaluated participants' behavioral performance in the listening task with respect to accuracy and response speed. For the binary measure of accuracy, we excluded trials in which participants failed to answer within the given 4-s response window ('timeouts'). Spatial stream confusions, that is trials in which the sentence-final word of the to-be-ignored speech stream were selected, and 
random errors were jointly classified as incorrect answers. The analysis of response speed, defined as the inverse of reaction time, was based on correct trials only. Single-trial behavioral measures were subjected to (generalized) linear mixed-effects analysis (see Statistical analysis).

\section{EEG data analysis}

\section{Time-frequency analysis}

To obtain time-frequency representations of single-trial oscillatory power, the preprocessed continuous EEG data were high-pass-filtered at $0.3 \mathrm{~Hz}$ (finite impulse response (FIR) filter, zero-phase lag, order 5574, Hann window) and lowpass-filtered at $180 \mathrm{~Hz}$ (FIR filter, zero-phase lag, order 100, Hamming window). The EEG was cut into epochs of -2 to $8 \mathrm{~s}$ relative to the onset of the spatialattention cue to capture cue presentation as well as the entire auditory stimulation interval. Individual epochs were convolved with frequency-adaptive Hann-tapers of 7 cycles width $(2-30 \mathrm{~Hz}$, step size $0.5 \mathrm{~Hz}$ ). Oscillatory power was estimated by squaring the magnitude of the complex-valued Fourier coefficients at each single frequency. Since oscillatory power or amplitude values typically follow a highly skewed, non-normal distribution, we applied a nonlinear transformation of the Box-Cox family (power $r_{\text {trans }}=\left(\right.$ power $\left.^{p}-1\right) / p$ with $\left.p=0.2\right)$ to minimize skewness and to satisfy the assumption of normality for parametric statistical tests involving oscillatory power values (Smulders et al., 2018).

To capture whole-trial (0-7 s) dynamics in oscillatory power across all trials, we first subtracted power averaged across trials in this interval from individual trials (absolute baseline). Grand average representations of oscillatory power were obtained by averaging the result across all trials and participants.

\section{Attentional modulation of alpha power}

To quantify attention-related changes in $8-12 \mathrm{~Hz}$ alpha power, we calculated two attentional modulation indices separately for selective- and dividedattention trials: the topographical alpha lateralization index $\left[A L I_{\text {topo }}=\left(\alpha-\right.\right.$ power $_{\text {att- }}$ left $-a^{-}$power $\left._{\text {att-right }}\right)$ / (a-power att-left $+a-$ power $\left.\left._{\text {att-right }}\right)\right]$ and the single-trial, temporally resolved alpha lateralization index $\left[\mathrm{ALI}=\right.$ (a-power $_{\text {ipsi }}-\mathbf{a}$ power $\left._{\text {contra }}\right) /\left(a-\right.$ power $_{\text {ipsi }}+$ a-power $\left._{\text {contra }}\right)$ ] (Haegens et al., 2011; Wöstmann et al., 2016; Tune et al., 2018).

The $A \mathrm{Al}_{\mathrm{topo}}$, calculated per channel for $8-12 \mathrm{~Hz}$ absolute alpha power, provides a spatial representation of attentional effects. Positive $\mathrm{ALI}_{\text {topo }}$ values reflect higher alpha power levels during attend-left compared to attend-right trials. The reverse relation holds for negative values. For divided attention trials in which attention was not cued to one of the two sides, trials were categorized based on the probed ear.

In estimating the $A L I_{\text {topo }}$ we used a robust variant of the index that applies the inverse logit transform $[(1 / 1+\exp (-x)]$ to both inputs to scale them into a common, positive-only [0;1]-bound space prior to index calculation.

To calculate the ALI, which provides time-resolved single-trial estimates of the attentional modulation of alpha power, we first corrected for overall hemispheric power differences independent of attentional modulation. To this end, we normalized single-trial power values by calculating per channel and frequency the whole-trial $(0-7 \mathrm{~s})$ power averaged across all trials and subtracting 
884 it from single trials (see Fig. 3-supplement 2 for ALI calculated on absolute

We then selected per participant the ten channels with the largest positive $A \mathrm{LI}_{\text {topo }}$ values over the left posterior hemisphere and the ten channels with the largest negative $A \mathrm{LI}_{\text {topo }}$ values over the right posterior hemisphere. For each of the two attention conditions (attend-/probed-left versus attend/probed-right), the selected channels were classified as being either ipsi- or contralateral to the focus of attention (in divided-attention trials: probed ear). To obtain a time-resolved measure of attentional modulation of alpha power, we calculated the ALI over the time course of the entire trial using a 250-ms sliding window (Mesgarani and Chang, 2012). Index calculation applied the same inverse logit transformation as used for the $\mathrm{ALI}_{\text {topo. }}$. For statistical analysis of cuedriven neural modulation, we extracted single-subject mean ALI values per experimental condition in the time window of sentence presentation (3.5-6.5 s), and treated them as the dependent measure in linear mixed-effects analysis. In addition, they served as continuous predictors in the statistical analysis of brainbehavior relationships (see below).

assess the neural encodir

903 To assess the neural encoding of speech by low-frequency activity, the 904 continuous preprocessed EEG were down-sampled to $f_{s}=125 \mathrm{~Hz}$, filtered 905 between $f_{c}=1$ and $8 \mathrm{~Hz}$ (FIR filters, zero-phase lag, order: $8 f_{s} / f_{c}$ and $2 f_{s} / f_{c}$, 906 Hamming window), and re-referenced to the average of both mastoid 907 electrodes (TP9 and TP10). The EEG was cut to yield individual epochs covering 908 the presentation of auditory stimuli, beginning at noise onset until the end of 909 auditory presentation. Individual epochs were z-scored prior to submitting them to regression analysis (see below).

913 From the presented speech signals, we derived a temporal representation of the 914 acoustic onsets in the form of the onset envelope (cf. Fiedler et al., 2019). To this 915 end, using the NSL toolbox (Chi et al., 2005), we first extracted an auditory 916 spectrogram of the auditory stimuli (128 spectrally resolved sub-band envelopes 917 logarithmically spaced between $90-4000 \mathrm{~Hz}$ ), which were then summed across 918 frequencies to yield a broad-band temporal envelope. Next, the output was 919 down-sampled and low-pass filtered to match the specifics of the EEG. To derive 920 the final onset envelope to be used in linear regression, we first obtained the 921 first derivative of the envelope and set negative values to zero (half-wave 922 rectification) to yield a temporal signal with positive-only values reflecting the 923 acoustic onsets (see Fig. 4A and figure supplements).

\section{Estimation of envelope reconstruction models}

926 To investigate how low-frequency (i.e, $<8 \mathrm{~Hz}$ ) fluctuations in EEG activity relate 927 to the encoding of attended and ignored speech, we used a linear regression 928 approach to describe the mapping from the presented speech signal to the 929 resulting neural response (Lalor and Foxe, 2010; Crosse et al., 2016). More 930 specifically, we trained stimulus reconstruction models (also termed decoding or 931 backward models) to predict the onset envelope of the attended and ignored 
Neural tracking and alpha power in attentive listening

932 speech stream from EEG. In this analysis framework, a linear reconstruction 933 model $g$ is assumed to represent the linear mapping from the recorded EEG 934 signal, $r(t, n)$, to the stimulus features, $s(t)$ :

where $s^{\wedge}(t)$ is the reconstructed onset envelope at time point $t$. We used all 64 EEG channels and time lags $\tau$ in the range of $-100 \mathrm{~ms}$ to $500 \mathrm{~ms}$ to compute envelope reconstruction models using ridge regression (Hoerl and Kennard, 1970):

where $R$ is a matrix containing the sample-wise time-lagged replication of the neural response matrix $r, \lambda$ is the ridge parameter for regularization, $m$ is a scalar representing the mean of the trace of $R^{\top} R$ (Biesmans et al., 2016), and $I$ is the identity matrix. We followed the procedures described in (Fiedler et al., 2017) to estimate the optimal ridge parameter at $\lambda=5$.

Compared to linear forward ('encoding') models that derive temporal response functions (TRFs) independently for each EEG channel, stimulus reconstruction models represent multivariate impulse response functions that exploit information from all time lags and EEG channels simultaneously. To allow for a neurophysiological interpretation of backward model coefficients, we additionally transformed them into linear forward model coefficients following the inversion procedure described in Haufe et al. (2014). All analyses were performed using the multivariate temporal response function (mTRF) toolbox (version 1.5; Crosse et al., 2016) for Matlab.

Prior to model estimation, we split the data based on the two spatial attention conditions (selective vs. divided), resulting in 120 trials per condition.

961 Envelope reconstruction models were trained on selective-attention single-trial 962 data, only. Two different backward models were estimated for a given trial, an 963 envelope reconstruction model for the-be-attended speech stream (short: 964 attended reconstruction model), and one for the to-be-ignored speech stream 965 (short: ignored reconstruction model). Reconstruction models for attended and 966 ignored speech signals were trained separately for attend-left and attend-right 967 trials which yielded 120 single-trial decoders (60 attended, 60 ignored) per 968 attentional setting. For illustrative purposes and to evaluate decoding accuracy, 969 we averaged the reconstruction models for attended and ignored envelopes 970 and their forward transformations across left and right trials for all participants 971 (Fig. 4B).

972

974 To quantify how strongly the attended and ignored sentences were tracked by 975 slow cortical dynamics, at the single-subject level, we reconstructed the 976 attended and ignored envelope of a given trial using a leave-one-out cross977 validation procedure. Following this approach, the envelopes of each trial were 978 reconstructed using the averaged reconstruction models trained on all but the 979 tested trial. For a given trial, we only used the trained models that corresponded 
980 to the respective cue condition (i.e., for an attend-left/ignore-right trial we only 981 used the reconstruction models trained on the respective trials). The 982 reconstructed onset envelope obtained from each model was then compared to 983 the two onset envelopes of the actually presented speech signals. The resulting 984 Pearson-correlation coefficients, $r_{\text {attended }}$ and $r_{\text {ignored, }}$, reflect the single-trial neural 985 tracking strength or reconstruction accuracy (cf. O'Sullivan et al., 2014; see also 986 Fig. 4-supplement 1).

We proceeded in a similar fashion for divided-attention trials. Since these trials could not be categorized based on the to-be-attended and -ignored side, we split them based on the ear that was probed at the end the trial. Given that even in the absence of a valid attention cue, participants might still (randomly) focus their attention to one of the two streams, we wanted to quantify how strongly the probed and unprobed envelopes were tracked neurally. To this end, we used the reconstruction models trained on selectiveattention trials to reconstruct the onset envelopes of divided-attention trials. Sentences presented in probed-left/unprobed-right trials were reconstructed using the attend-left/ignore-right reconstruction models while probedright/unprobed-left trials used the reconstruction models trained on attendright/ignore-left trials.

One of the goals of the present study was to investigate the degree to which the neural tracking of the attended and ignored envelopes would be influenced by spatial attention and semantic predictability, and how these changes would relate to behavior. We thus used the neural tracking strength of the attended envelope (when reconstructed with the attended reconstruction model) and that of the ignored envelope (when reconstructed with the ignored reconstruction model) as (in-)dependent variables in our linear mixed-effects analyses (see below).

\section{Decoding accuracy}

1010 To evaluate how accurately we could decode an individual's focus of attention, we separately evaluated the performance of the reconstruction models for the

1012 the attended reconstruction models, the focus of attention was correctly

1013 decoded if the correlation with the attended envelope was greater than that

1014 with the ignored envelope. Conversely, for reconstructions performed with the 1015 ignore reconstruction models, a correct identification of attention required the 1016 correlation with the ignored envelope to be greater than that with the attended 1017 envelope (see Fig. 4-supplement 1). To quantify whether the single-subject 1018 decoding accuracy was significantly above chance, we used a binomial test with $\mathrm{a}=0.05$.

1020

\section{Statistical analysis}

1022 We used (generalized) linear mixed-effect models to investigate the influence of 1023 the experimental cue conditions (spatial cue: divided vs. selective; semantic cue: 1024 general vs. specific) on behavior, see Q1 in Fig. 1) as well as on our neural 1025 measures of interest (Q2). Finally, we investigated the relationship of neural 1026 measures (Q3) and their (joint) influence of the different cue conditions and 1027 neural measures on behavior (Q4). Using linear mixed-effects models allowed us 
1028 to model and control for the impact of various additional covariates known to 1029 influence behavior and/or neural measures. These included the probed ear 1030 (left/right), whether the later-probed sentence had the earlier onset (yes/no), as 1031 well as participants' age and hearing acuity (pure-tone average across both 1032 ears).

1033 Model estimation and selection followed an iterative model fitting 1034 procedure that started with an intercept-only null model (Tune et al., 2018). 1035 Fixed effects terms were added in a stepwise procedure in the order of their 1036 conceptual importance for the tested hypotheses, beginning with main effects 1037 followed by higher-order interactions, and the change of model fit (fitted using 1038 maximum-likelihood estimation) was assessed using likelihood ratio tests. The 1039 effects included in the brain-behavior model were guided by effects found in 1040 the cue-driven neural modulation models. Deviation coding was used for 1041 categorical predictors. All continuous variables were z-scored.

1042 For the dependent measure of accuracy, we used generalized linear 1043 mixed-effects model (binomial distribution, logit link function). For response 1044 speed, we used general linear mixed-effects model (gaussian distribution, 1045 identity link function). $P$-values for individual model terms in the linear models 1046 are based on $t$-values and use the Satterthwaite approximation for degrees of 1047 freedom (Luke, 2017). P-values for generalized linear models are based on z1048 values and asymptotic Wald tests. Post hoc linear contrasts for categorical 1049 regressors with more than two levels were carried out on predicted marginal 1050 means ('least-square means') and standard errors estimated from the model 1051 (Lenth, 2016). In lieu of a standardized measure of effect size for mixed-effects 1052 models, we report odds ratios (OR) for generalized linear models and 1053 standardized regression coefficients $(\beta)$ for linear models.

All analyses were performed in R (R Core Team, 2018) using the packages

1055

1056

\section{Data availability}

1058 The complete dataset associated with this work including raw data, EEG data 1059 analysis results, as well as corresponding code will be publicly available under 1060 https://osf.io/28r57/. 
bioRxiv preprint doi: https://doi.org/10 1101/512251; this version posted January 4, 2019. The copyright holder for this preprint (which was not certified by peer review) is the author/funder, who has granted bioRxiv a license to display the preprint in perpetuity. It is made available under aCC-BY-NC-ND 4.0 International license.

Neural tracking and alpha power in attentive listening

\section{Acknowledgement}

1062 Felix Deilmann, Elisabeth Ni, Franziska Scharata, Philipp Seidel, and Annika 1063 Struck helped acquire and manage the data. Research was supported by the

1064 European Research Council (ERC Consolidator grant AUDADAPT, no. 646696) to

1065 JO. 
Neural tracking and alpha power in attentive listening

1066

1067

1068

1069

1070

1071

1072

1073

1074

1075

1076

1077

1078

1079

1080

1081

1082

1083

1084

1085

1086

1087

1088

1089

1090

1091

1092

1093

1094

1095

1096

1097

1098

1099

1100

1101

1102

1103

1104

\section{References}

Ahveninen J, Huang S, Belliveau JW, Chang W-T, Hämäläinen M (2013) Dynamic oscillatory processes governing cued orienting and allocation of auditory attention. J Cogn Neurosci 25:1926-1943.

Alavash M, Tune S, Obleser J (2018) Modular reconfiguration of an auditorycontrol brain network supports adaptive listening behavior. Proc Natl Acad Sci USA 48:201815321-10.

Banerjee S, Snyder AC, Molholm S, Foxe JJ (2011) Oscillatory Alpha-Band Mechanisms and the Deployment of Spatial Attention to Anticipated Auditory and Visual Target Locations: Supramodal or Sensory-Specific Control Mechanisms? J Neurosci 31:9923-9932.

Broadbent DE, Gregory M (1964) Accuracy of recognition for speech presented to the right and left ears. Quarterly Journal of Experimental Psychology 16:359-360.

Chait M, de Cheveigné A, Poeppel D, Simon JZ (2010) Neural dynamics of attending and ignoring in human auditory cortex. Neuropsychologia 48:3262-3271.

Cherry EC (1953) Some experiments on the recognition of speech, with one and with two ears. J Acoust Soc Am 25:975-979.

Chi T, Ru P, Shamma SA (2005) Multiresolution spectrotemporal analysis of complex sounds. J Acoust Soc Am 118:887-906.

Crosse MJ, Butler JS, Lalor EC (2015) Congruent Visual Speech Enhances Cortical Entrainment to Continuous Auditory Speech in Noise-Free Conditions. J Neurosci 35:14195-14204.

Crosse MJ, Di Liberto GM, Bednar A, Lalor EC (2016) The Multivariate Temporal Response Function (mTRF) Toolbox: A MATLAB Toolbox for Relating Neural Signals to Continuous Stimuli. Front Hum Neurosci 10:604.

Delorme A, Makeig S (2004) EEGLAB: an open source toolbox for analysis of single-trial EEG dynamics including independent component analysis. Journal of Neuroscience Methods 134:9-21.

Ding N, Simon JZ (2012) Emergence of neural encoding of auditory objects while listening to competing speakers. Proc Natl Acad Sci USA 109:1 1854-11859.

Ding N, Simon JZ (2014) Cortical entrainment to continuous speech: functional roles and interpretations. Front Hum Neurosci 8:13367.

Bates D, Mächler M, Bolker B, Walker S (2015) Fitting Linear Mixed-Effects Models Using Ime4. J Stat Soft 67:1-48.

Etard O, Kegler M, Braiman C, Forte AE, Reichenbach T (2018) Real-time decoding of selective attention from the human auditory brainstem response to continuous speech. bioRxiv doi: 10.1101/259853 
Neural tracking and alpha power in attentive listening

1105 Fiedler L, Wöstmann M, Graversen C, Brandmeyer A, Lunner T, Obleser J (2017)

1106

1107

1108

1109

1110

1111

1112

1113

1114

1115

1116

1117

1118

1119

1120

1121

1122

1123

1124

1125

1126

1127

1128

1129

1130

1131

1132

1133

1134

1135

1136

1137

1138

1139

1140

1141

1142

1143

Single-channel in-ear-EEG detects the focus of auditory attention to concurrent tone streams and mixed speech. Journal of Neural Engineering 14: 036020 .

Fiedler L, Wöstmann M, Herbst SK, Obleser J (2019) Late cortical tracking of ignored speech facilitates neural selectivity in acoustically challenging conditions. Neurolmage 186:33-42.

Foxe JJ, Snyder AC (2011) The role of alpha-band brain oscillations as a sensory suppression mechanism during selective attention. Front Psychology 2:154.

Giraud AL, Poeppel D (2012) Cortical oscillations and speech processing: emerging computational principles and operations. Nat Neurosci 15:511517.

Haegens S, Händel BF, Jensen O (2011) Top-down controlled alpha band activity in somatosensory areas determines behavioral performance in a discrimination task. J Neurosci 31:5197-5204.

Hamilton LS, Huth AG (2018) The revolution will not be controlled: neural stimuli in speech neuroscience. Lang Cogn Neurosci 27:1-10.

Haufe S, Meinecke F, Görgen K, Dähne S, Haynes J-D, Blankertz B, Bießmann F (2014) On the interpretation of weight vectors of linear models in multivariate neuroimaging. Neurolmage 87:96-110.

Händel BF, Haarmeier T, Jensen O (2011) Alpha oscillations correlate with the successful inhibition of unattended stimuli. J Cogn Neurosci 23:2494-2502.

Henry MJ, Herrmann B, Kunke D, Obleser J (2017) Aging affects the balance of neural entrainment and top-down neural modulation in the listening brain. Nat Commun 8:15801.

Henry MJ, Obleser J (2012) Frequency modulation entrains slow neural oscillations and optimizes human listening behavior. Proc Natl Acad Sci USA 109:20095-20100.

Hoerl AE, Kennard RW (1970) Ridge Regression: Biased Estimation for Nonorthogonal Problems. Technometrics 12:55-67.

Hong X, Sun J, Bengson JJ, Mangun GR, Tong S (2015) Normal aging selectively diminishes alpha lateralization in visual spatial attention. Neurolmage 106:353-363.

Horton C, D'Zmura M, Srinivasan R (2013) Suppression of competing speech through entrainment of cortical oscillations. J Neurophysiol 109:3082-3093.

Jefferies K, Gale TM (2013) 6-CIT: Six-Item Cognitive Impairment Test. In: Cognitive Screening Instruments, pp 209-218. London: Springer, London.

Jensen O, Mazaheri A (2010) Shaping functional architecture by oscillatory alpha activity: gating by inhibition. Front Hum Neurosci 4: 186. 
Neural tracking and alpha power in attentive listening

1144 Kaya EM, Elhilali M (2017) Modelling auditory attention. Philosophical

1145 Transactions of the Royal Society B: Biological Sciences 372: 1714.

1146 Kerlin JR, Shahin AJ, Miller LM (2010) Attentional gain control of ongoing cortical

Kimura D (1961) Cerebral dominance and the perception of verbal stimuli. Canadian Journal of Psychology/Revue canadienne de psychologie 15:166171.

Krakauer JW, Ghazanfar AA, Gomez-Marin A, Maclver MA, Poeppel D (2017) Neuroscience needs behavior: correcting a reductionist bias. Neuron 93:480-490.

Lakatos P, Barczak A, Neymotin SA, McGinnis T, Ross D, Javitt DC, O'Connell MN (2016) Global dynamics of selective attention and its lapses in primary auditory cortex. Nat Neurosci 19:1707-1717.

Lalor EC, Foxe JJ (2010) Neural responses to uninterrupted natural speech can be extracted with precise temporal resolution. Eur J Neurosci 31:189-193. lateralization during working memory but not during attentional cueing in older adults. Cereb Cortex 28:21-32.

Lenth RV (2016) Least-squares means: The R package Ismeans. J Stat Soft 69:133.

Luke SG (2017) Evaluating significance in linear mixed-effects models in R. Behav Res 49:1494-1502.

Lüdecke D (2018) Data Visualization for Statistics in Social Science [R package sjPlot version 2.6.1]. Available at: https://CRAN.Rproject.org/package $=$ sjPlot.

Melara RD, Rao A, Tong Y (2002) The duality of selection: Excitatory and inhibitory processes in auditory selection attention. Journal of Experimental Psychology: Human Perception and Performance 28:279-306.

Mesgarani N, Chang EF (2012) Selective cortical representation of attended speaker in multi-talker speech perception. Nature 485:233-236.

Mok RM, Myers NE, Wallis G, Nobre AC (2016) Behavioral and neural markers of flexible attention over working memory in aging. Cereb Cortex 26:18311842.

1177 Müller N, Weisz N (2011) Lateralized auditory cortical alpha band activity and 1178 interregional connectivity pattern reflect anticipation of target sounds. 1179 Cereb Cortex 22:1604-1613.

1180 Nourski KV, Brugge JF (2011) Representation of temporal sound features in the 1181 human auditory cortex. Rev Neurosci 22:187-203.

1182 Obleser J, Weisz N (2012) Suppressed Alpha Oscillations Predict Intelligibility of 
Neural tracking and alpha power in attentive listening Speech and its Acoustic Details. Cereb Cortex 22:2466-2477.

Oldfield RC (1971) The assessment and analysis of handedness: The Edinburgh inventory. 9:97-113.

O'Sullivan JA, Power AJ, Mesgarani N, Rajaram S, Foxe JJ, Shinn-Cunningham BG, Slaney M, Shamma SA, Lalor EC (2014) Attentional Selection in a Cocktail Party Environment Can Be Decoded from Single-Trial EEG. Cereb Cortex 25:1697-1706.

Peelle JE, Gross J, Davis MH (2013) Phase-Locked Responses to Speech in Human Auditory Cortex are Enhanced During Comprehension. Cereb Cortex 23:1378-1387.

Peirce JW (2007) PsychoPy—Psychophysics software in Python. Journal of Neuroscience Methods 162:8-13.

Posner MI (1980) Orienting of attention. Quarterly Journal of Experimental Psychology 32:3-25.

Power AJ, Foxe JJ, Forde E-J, Reilly RB, Lalor EC (2012) At what time is the cocktail party? A late locus of selective attention to natural speech. Eur J Neurosci 35:1497-1503.

Presacco A, Simon JZ, Anderson S (2016a) Effect of informational content of noise on speech representation in the aging midbrain and cortex. J Neurophysiol 116:2356-2367.

Presacco A, Simon JZ, Anderson S (2016b) Evidence of degraded representation of speech in noise, in the aging midbrain and cortex. J Neurophysiol 116:2346-2355.

R Core Team (2018) R: A language and environment for statistical computing. Available at: https://www.R-project.org/.

Rihs TA, Michel CM, Thut G (2007) Mechanisms of selective inhibition in visual spatial attention are indexed by a-band EEG synchronization. Eur J Neurosci 25:603-610.

Sadaghiani S, Kleinschmidt A (2016) Brain Networks and a-Oscillations: Structural and Functional Foundations of Cognitive Control. Trends Cogn Sci 20:805-817.

Sander MC, Werkle-Bergner M, Lindenberger U (2012) Amplitude modulations and inter-trial phase stability of alpha-oscillations differentially reflect working memory constraints across the lifespan. Neurolmage 59:646-654.

Schroeder CE, Lakatos P (2009) Low-frequency neuronal oscillations as instruments of sensory selection. Trends Neurosci 32:9-18.

Schroeder CE, Wilson DA, Radman T, Scharfman H, Lakatos P (2010) Dynamics of Active Sensing and perceptual selection. Current Opinion in Neurobiology 20:172-176. 
Neural tracking and alpha power in attentive listening

1222 Shinn-Cunningham BG (2008) Object-based auditory and visual attention. Trends Cogn Sci 12:182-186.

1224 Shinn-Cunningham BG, Best V (2008) Selective Attention in Normal and Impaired Hearing. Trends in Amplification 12:283-299.

Smulders FTY, Oever ten S, Donkers FCL, Quaedflieg CWEM, van de Ven V (2018) Single-trial log transformation is optimal in frequency analysis of resting EEG alpha. Eur J Neurosci 44:94-14.

Sohoglu E, Peelle JE, Carlyon RP, Davis MH (2012) Predictive Top-Down Integration of Prior Knowledge during Speech Perception. J Neurosci 32:8443-8453.

Tune S, Wöstmann M, Obleser J (2018) Probing the limits of alpha power lateralisation as a neural marker of selective attention in middle-aged and older listeners. Eur J Neurosci 25:1926-14.

van Ede F, Köster M, Maris E (2012) Beyond establishing involvement: quantifying the contribution of anticipatory $\alpha$ - and $\beta$-band suppression to perceptual improvement with attention. J Neurophysiol 108:2352-2362.

Wöstmann M, Herrmann B, Maess B, Obleser J (2016) Spatiotemporal dynamics of auditory attention synchronize with speech. Proc Natl Acad Sci USA

$1240 \quad 113: 3873-3878$.

1241 Wöstmann M, Lim S-J, Obleser J (2017) The human neural alpha response to 1242 speech is a proxy of attentional control. Cereb Cortex 27:3307-3317.

1243 Wöstmann M, Obleser J (2016) Acoustic detail but not predictability of taskirrelevant speech disrupts working memory. Front Hum Neurosci 10:201209.

Wöstmann M, Vosskuhl J, Obleser J, Herrmann CS (2018) Opposite effects of lateralised transcranial alpha versus gamma stimulation on auditory spatial attention. Brain Stimulation: 11:752-758.

Zion Golumbic EMZ, Ding N, Bickel S, Lakatos P, Schevon CA, McKhann GM, Goodman RR, Emerson R, Mehta AD, Simon JZ, Poeppel D, Schroeder CE (2013) Mechanisms Underlying Selective Neuronal Tracking of Attended Speech at a "Cocktail Party". Neuron 77:980-991. 